\title{
Mesenchymal Stem Cells: A Potential Therapeutic Strategy for Neurodegenerative Diseases
}

\author{
Panpan Yao Liping Zhou Lujie Zhu Binjie Zhou Qin Yu \\ College of Life Science, Zhejiang Chinese Medical University, Hangzhou, PR China
}

\section{Keywords}

Mesenchymal stem cells · Neurodegenerative diseases .

Mechanism · Clinical treatment

\begin{abstract}
Neurodegenerative disease is a kind of chronic, progressive nervous system disease characterized by neuron degeneration or apoptosis. Current treatments cannot prevent the development of the disease. Possible alternative treatments include cell therapy, especially with the use of mesenchymal stem cells (MSCs). MSCs are pluripotent stem cells with capacities for self-renewal and multidirectional differentiation. MSCs may serve as a reliable source of neural cells for potential cell replacement therapy or regenerative medicine treatment. Here, we summarized the therapeutic mechanisms of MSCs and how they can contribute to the development of treatments for neurodegenerative diseases.
\end{abstract}

(c) 2020 S. Karger AG, Basel

\section{Introduction}

Neurodegenerative diseases are common neurological diseases that are characterized by the large loss of specific neurons; these diseases include Parkinson's disease (PD), Alzheimer's disease (AD), and amyotrophic lateral scle-

karger@karger.com

(c) 2020 S. Karger AG, Basel

www.karger.com/ene

Karger" rosis (ALS). A key pathological feature of these diseases is the accumulation of misfolded proteins in the brain, leading to neurological dysfunction and disease [1]. Although the aetiology of this disease has been proven to be related to a variety of cellular and molecular mechanisms, the pathogenesis remains unclear, and the pathogenic factors are complex. For most patients, early diagnosis is hindered due to the lack of effective biomarkers [2]. The development of neuronal degeneration is accompanied by secondary effects that impair the quality of life. The available treatments can delay the development of the disease, but they fail to achieve satisfactory results and lead to poor prognoses.

In recent decades, scientists have made great efforts in the treatment of neurodegenerative diseases. Due to the limited ability of brain self-repair and regeneration, the traditional treatment of neurodegenerative diseases has not achieved ideal results. Stem cells, which have strong differentiation capacity, are considered the best potential therapy for brain tissue damage or degeneration caused by injury, ageing, or disease. In recent years, with advances in the understanding and development of stem cells and related technologies, the treatment of neurodegenerative diseases has ushered in new opportunities, and

Panpan Yao and Liping Zhou contributed equally to this work.

Qin Yu

College of Life Science, Zhejiang Chinese Medical University

548 Binwen Road

Hangzhou, Zhejiang 310053 (PR China)

qinyu3587@126.com 


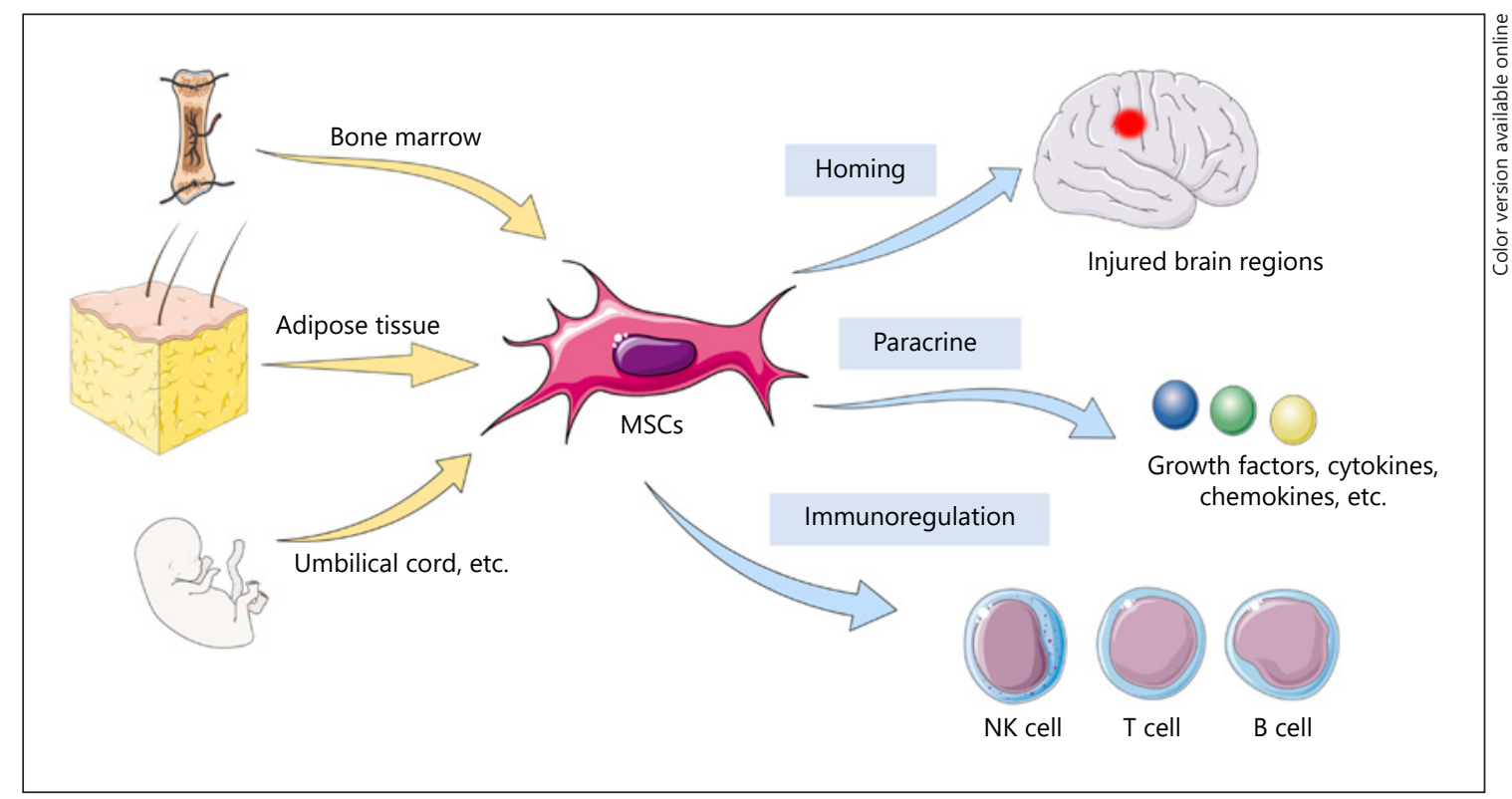

Fig. 1. MSCs mainly derived from bone marrow, adipose tissue, and umbilical cord. Putative mechanisms of MSCs in the treatment of neurodegenerative diseases: (1) homing to the injured brain regions, (2) paracrine neuroprotective factors, and (3) regulate the function of immune cells. MSCs, mesenchymal stem cells.

people have begun to treat neurodegenerative diseases with cell-based therapies.

Mesenchymal stem cells (MSCs) are adult stem cells with potential for self-renewal and multi-lineage differentiation [3], and they can differentiate into a variety of mesenchymal tissues, such as bone, cartilage, adipose, and haematopoietic tissue [4]. MSCs originate from the mesoderm, but studies have shown that MSCs can be horizontally differentiated into non-mesoderm-derived cells, such as glial cells and neurons $[5,6]$, which can be considered an ideal source for replacing lost cells in neurodegenerative diseases. In addition, MSCs are easy to collect, isolate, and culture; have low immunogenicity and immunomodulatory functions; and have immunoregulatory properties. Therefore, MSCs are promising as treatments for neurodegenerative diseases.

\section{Mechanisms of MSCs in the Treatment of Neurodegenerative Diseases}

The therapeutic mechanisms of MSCs in the treatment of neurodegenerative diseases are still being explored, although studies have shown that MSCs may improve the disease in the following ways (Fig. 1).

\section{Homing}

Homing refers to the characteristic that MSCs can spontaneously migrate to the injured region when the body is damaged. Homing ability, first demonstrated in MSCs by Saito et al. [7], is an important characteristic of MSCs that can be widely used in disease treatment. Chopp et al. [8] demonstrated that, after implantation of BrdUlabelled MSCs into rats with spinal cord injury, MSCs could migrate to the injury side and differentiate into neurons and glial cells, leading to persisting improvement of motor function. Many studies have found that transplanted exogenous MSCs preferentially home to inflamed areas and injured tissue upon damage. Ischaemic tissue can attract the homing of MSCs, and homing to the injured site can play a therapeutic role [9]. However, a growing number of studies have shown that MSCs home to a low proportion of tissues in vivo [10], which is related to transplantation efficacy. The effective homing of MSCs can be influenced by a variety of factors, such as the time and quantity of transplantation, the culture method, pretreatment, and the transplantation method of MSCs [11-13]. Therefore, how to improve the homing efficiency of MSCs is an urgent problem to be solved.

MSCs have been proven to have the ability to home to injured areas after transplantation, which means that they are very promising as therapeutic drug carriers. Drugs 
entering the central nervous system should be able to pass through the blood-brain barrier and target specific cell types in different central nervous system regions, which requires a carrier that can efficiently deliver the drug to the target location. MSCs can be used as vehicles for drug delivery, which can provide an effective platform for targeted treatment in neurodegenerative diseases.

\section{Paracrine}

MSCs can secrete a series of growth factors, cytokines, chemokines, and various enzymes, playing an important role in cell migration and immune regulation [14]. Hoban et al. [15] implanted MSCs overexpressing glial cell linederived neurotrophic factor into the striatum of PD model rats. TH positive staining was found directly proximal to the transplant site, indicating that glial cell line-derived neurotrophic factor has neurotrophic capability in the PD model. MSCs can also secrete exosomes, which are rich in endosomal-derived components and bioactive molecules, such as proteins, lipids, mRNA, tRNA, genomic DNA, and mitochondrial DNA [16-18]. Exosomes can inhibit inflammation, participate in immune responses and antigen presentation, and promote cell differentiation and migration. Exosomes secreted by MSCs have attracted much attention in regenerative medicine due to their unique ability to repair damaged tissues. It has been reported that MSC-derived exosomes can be used as a direct therapeutic agent, which can effectively inhibit inflammation after traumatic spinal cord injury and the activation of $\mathrm{A} 1$ neurotoxic reactive astrocytes [19]. Studies have shown that MSC-derived exosomes stimulate neurogenesis in the subventricular zone and reduce cognitive impairment in a mouse model of AD [20]. Pegtel et al. [21] injected exosomes co-cultured with MSCs and neurons into the mouse model of muscle atrophy, and this effectively promoted the recovery of astrocytes and, thus, improved symptoms. Therefore, MSCderived exosomes are expected to be a promising clinical treatment strategy.

\section{Immunoregulation}

MSCs are undifferentiated precursor stem cells with immature phenotypic differentiation and low immunogenicity. The immunoregulation of MSCs may be related to their interaction with T cells, B cells, and natural killer cells (NK cells). MSCs can regulate the function of immune cells by suppressing the production of antibodies by $\mathrm{B}$ cells, the activation of $\mathrm{T}$ cells, and the secretion of cytokines by NK cells [22]. MSCs can avoid recognition and dissolution when co-cultured with allogeneic cyto-

MSCs in Treatment of Neurodegenerative Diseases toxic T lymphocytes and NK cells [23]. Sotiropoulou et al. [24] suggested that the proliferation of NK cells could be suppressed by the reaction between MSCs and NK cells and the secretion of soluble molecules by MSCs. In addition, when adipose-derived MSCs (AD-MSCs) were cocultured with B cells, AD-MSCs could suppress B-cell proliferation and transfer the cytokine spectrum of $\mathrm{B}$ cells to the anti-inflammatory spectrum [25]. MSCs can also inhibit T-cell proliferation and co-regulate cytokine receptor expression by cell contact [26]. Studies have found that placental-derived MSCs have beneficial immunosuppressive effects in $\mathrm{AD}$ mice. Animal experiments have shown that the immunoregulation of placental-derived MSCs can inhibit the release of inflammatory cytokines, alleviate cognitive impairment, and increase the survival rate of neurons and nerve regeneration [27]. Recently, immunosuppressants have been used in combination with MSCs [28-31], and this is expected to be used in the clinical treatment of neurodegenerative diseases in the future.

\section{MSCs in the Treatment of Neurodegenerative Diseases}

\section{Parkinson's Disease}

$\mathrm{PD}$ is a common neurodegenerative disease in ageing that can be characterized by tremor, myotonia, and reduced movement [32]. The main lesions of PD are in the substantia nigra and striatum. Due to the degeneration and death of dopamine (DA) neurons in the brain, the DA produced by the striatum is reduced. Currently, drug therapy is the main treatment for PD, including anticholinergics, dopaminergic agonists, and neuroprotectants. However, nonmotor symptoms, such as cognitive impairment, neuropsychiatric symptoms, and sleep disorders, cannot be improved by drugs.

Studies have shown that abnormally aggregated a-synuclein in the brain of PD patients can be recognized and attacked by the immune system as an antigen [33]. The patients have elevated levels of multiple inflammatory cytokines. The role of MSCs in cell migration and immune regulation will gain more advantages in the treatment of these diseases. In addition, MSCs can differentiate into neuron-like cells under specific culture conditions. Mostafavi et al. [34] transplanted green fluorescent protein-labelled trabecular meshwork MSCs into a rat model of PD. The results showed that the motor performance was significantly improved, and trabecular meshwork MSCs have the potential to differentiate into 
DA neurons. Similar results were obtained by transplanting human adult MSCs into the striatum of rats lesioned with 6-hydroxydopamine [35]. In addition, MSCs can inhibit the polarization of microglia cells, reduce the level of $\alpha$-synuclein, and promote the survival of neurons in mice [36]. These data suggest that MSCs may play a role in cell replacement, anti-inflammatory, and immune regulation in PD treatment [37]. Furthermore, the use of MSCs with high potential of differentiation into DA cells appears to be a suitable strategy in cell therapy for PD. At present, several clinical trials of MSCs in the treatment of PD have been carried out, and further long-term effects need to be explored [38].

\section{Alzheimer's Disease}

$\mathrm{AD}$ is a common neurodegenerative disease with an insidious onset $[39,40]$. It is clinically characterized by cognitive decline, memory impairment, behavioural disorders, and aphasia [40]. It is believed that the pathological changes of AD include abnormal accumulation of amyloid $\beta$-protein $(A \beta)$ and excessive phosphorylation of Tau protein, forming plaque deposition and neurofibrillary tangles that result in central nervous system damage and microglia-mediated inflammatory responses [41]. However, the aetiology and pathogenesis of AD have not been elucidated, and there are no effective prevention or therapeutic methods. MSC-based cell therapy can support nerve growth by reconstructing neural microenvironment and reducing the factors that damage nerve structure, thus treating $\mathrm{AD}$.

In the brains of $\mathrm{AD}$ patients and model animals, overactivation of microglia and astrocytes can lead to inflammatory responses and cause neuronal damage. A previous study showed that the transplantation of BM-MSCs can activate endogenous microglia, promote the reduction of $A \beta$, and improve memory and cognitive functions [42]. In addition, human placental amniotic membranederived MSCs can improve the spatial learning and memory function of AD model mice. The number of amyloid plaques was also reduced, indicating that human placental amniotic membrane-derived MSC treatment might improve the pathology of $\mathrm{AD}$ and memory function through the regulation of oxidative stress [43]. Similarly, the transplantation of AD-MSCs can modulate microglial activation in $\mathrm{AD}$ mice, mitigate $\mathrm{AD}$ symptoms, and alleviate cognitive decline [44]. AD-MSCs can be easily harvested and are more abundant than other sources of MSCs. In one study, Park et al. [45] reported that AD-MSCs can migrate to the ageing brain, differentiate into neural cells, and enhance the physical and cog- nitive functions of aged mice by increasing acetylcholine levels and secreting growth and neurotrophic factors, such as brain-derived neurotrophic factor and nerve growth factor.

MSCs can also play a therapeutic role in the form of cell replacement. Experiments have shown that BMMSCs mobilized by granulocyte colony-stimulating factor can substitute impaired neurons, which may contribute to the recovery of cognitive function in $\mathrm{AD}$ model mice [46]. In addition, the role of cytokines emanating from MSCs and the signalling pathways involved in improving the conditions of neurodegenerative diseases have attracted substantial attention. Studies in this field have demonstrated that BM-MSCs co-cultured with A $\beta$ treated neural cells (as an in vitro cell line model for $\mathrm{AD}$ ) could significantly promote the secretion of cytokines and telomere length as a biomarker of neurodegenerative disorders as well as telomerase activity [47]. This study also showed a dramatic change in the gene and protein expression levels of important signalling pathways related to $\mathrm{AD}$, including mTOR, AMPK, GSK-3 $\beta$, and Wnt3/ $\beta$-catenin [47]. Nonetheless, further in vivo research is required to confirm the data. Such outcomes will pave the way towards simplified choices of well-suited cell sources to treat $\mathrm{AD}$.

\section{Amyotrophic Lateral Sclerosis}

ALS is a complex progressive neurodegenerative disease. Its occurrence is related to many pathological mechanisms, including mitochondrial dysfunction, oxidative stress, and axonal injury [48], which lead to degeneration and death of nerve cells. ALS occurs mostly in males [49] and develops rapidly. It is characterized by degeneration of both upper and lower motor neurons (MNs) in the brain and spinal cord, leading to progressive muscle atrophy and weakness in the limbs. Most patients die of respiratory disease within a few years after the onset of symptoms [49]. The available drugs can only relieve symptoms. ALS can be effectively treated if the survival time of MNs can be prolonged. Therapies based on MSCs may lead to more effective treatments for ALS.

Studies have shown that MSCs can migrate to the spinal cord of mice and suppress the activation of microglia and tissue glial proliferation, thus increasing the number of MNs, indicating that MSCs may have neuroprotective effects [50]. Experiments have shown that ASC-derived exosomes can protect MN-like NSC-34 cells from oxidative damage, increase cell survival [51], and reduce superoxide dismutase 1 aggregation and mitochondrial dysfunction [52]. 
Stem cell therapy based on neural growth factors (NTFs) secreted by MSCs has been used in clinical trials of ALS patients [53]. NTFs are critical material for the survival of neurons. In this study, MSC-NTFs could prolong the survival time of MNs in ALS patients. Injecting NTFs into the damaged neurons could improve the survival rate of ALS patients and slow the progress of disease. This therapy has demonstrated good safety and tolerance, but more clinical trials and scientific research are needed to optimize the treatment process to achieve the best effect.

\section{Conclusion}

MSCs have unique advantages in clinical application. As a kind of pluripotent stem cell, MSCs can differentiate into endoderm- and ectoderm-derived cells, such as glia and neurons, which provides a new approach for cell replacement therapy in neurodegenerative diseases [54]. In addition, MSCs have important clinical application value in nutrition, immunoregulation, and neuroprotection and can effectively avoid immune rejection and improve patients' quality of life.

Although numerous in vitro and in vivo experiments in animal models have confirmed the therapeutic potential of MSCs [55], the clinical results have not been encouraging. To date, there are more than 500 ongoing MSC-based clinical trials, and more than ten products have been approved for listing globally [56], indicating that their safety and effectiveness are being recognized. Studies have shown that no serious adverse reactions have been observed in animal and human clinical trials of MSCs (bone marrow, fat, umbilical cord, or placenta) from either autologous or allogeneic sources. No trans- plant-related tumour formation was found, and no transplant-related death occurred [57]. However, as with the development of other novel therapies, the application of MSCs will encounter unexpected events. It will be necessary to continuously explore safer, more effective and more feasible treatments. We need to optimize the process of preparing MSCs, the method of administration, and ideal dosages in order to provide more efficient treatment methods for patients. Further understanding the mechanisms will contribute to the therapeutic development of MSCs and further improve their potential therapeutic efficacy.

\section{Acknowledgements}

We thank the National Natural Science Foundation of China and Natural Science Foundation of Zhejiang Province.

\section{Conflict of Interest Statement}

The authors have no conflicts of interest to declare.

\section{Funding Sources}

This work was financially supported by the National Natural Science Foundation of China (grant number 31570994) and the Natural Science Foundation of Zhejiang Province (grant number LY15C100001).

\section{Author Contributions}

L.J.Z. conceived the idea. P.P.Y. and L.P.Z. designed the paper. B.J.Z. rivised the paper. Q.Y. guided the overall direction and improved the paper.

\section{References}

1 Chang YH, Wu KC, Harn HJ, Lin SZ, Ding DC. Exosomes and stem cells in degenerative disease diagnosis and therapy. Cell Transplant. 2018;27(3):349-63.

2 Nasello M, Schiro G, Crapanzano F, Balistreri CR. Stem cells and other emerging agents as innovative "drugs" in neurodegenerative diseases: benefits and limitations. Rejuvenation Res. 2018 Apr;21(2):123-40.

3 Dazzi F, Krampera M. Mesenchymal stem cells and autoimmune diseases. Best Pract Res Clin Haematol. 2011 Mar;24(1):49-57.

4 Choi JR, Yong KW, Choi JY. Effects of mechanical loading on human mesenchymal stem cells for cartilage tissue engineering. J Cell Physiol. 2018;233(3):1913-28.
5 Woodbury D, Schwarz EJ, Prockop DJ, Black IB. Adult rat and human bone marrow stromal cells differentiate into neurons. J Neurosci Res. 2000 Aug;61(4):364-70.

6 Dezawa M, Kanno H, Hoshino M, Cho H, Matsumoto N, Itokazu Y, et al. Specific induction of neuronal cells from bone marrow stromal cells and application for autologous transplantation. J Clin Invest. 2004;113(12): 1701-10.

7 Saito T, Kuang JQ, Bittira B, Al-Khaldi A, Chiu RC. Xenotransplant cardiac chimera: immune tolerance of adult stem cells. Ann Thora Surg. 2002 Jul;74(1):19-24.

8 Chopp M, Zhang XH, Li Y, Wang L, Chen J, $\mathrm{Lu} \mathrm{D}$, et al. Spinal cord injury in rat: treatment with bone marrow stromal cell transplantation. Neuroreport. 2000;11(13):3001-5.

9 Belema-Bedada F, Uchida S, Martire A, Kostin S, Braun T. Efficient homing of multipotent adult mesenchymal stem cells depends on FROUNT-mediated clustering of CCR2. Cell Stem Cell. 2008 Jun 5;2(6):566-75.

10 Karp JM, Leng Teo GS. Mesenchymal stem cell homing: the devil is in the details. Cell Stem Cell. 2009;4(3):206-16.

11 Chamberlain J, Yamagami T, Colletti E, Theise ND, Desai J, Frias A, et al. Efficient generation of human hepatocytes by the intrahepatic delivery of clonal human mesenchymal stem cells in fetal sheep. Hepatology. 2007;46(6):1935-45. 
12 Omori Y, Honmou O, Harada K, Suzuki J, Houkin K, Kocsis JD. Optimization of a therapeutic protocol for intravenous injection of human mesenchymal stem cells after cerebral ischemia in adult rats. Brain Res. 2008;1236: 30-8.

13 Fatimah SS, Tan GC, Chua K, Fariha MMN, Tan AE, Hayati AR. Stemness and angiogenic gene expression changes of serial-passage human amnion mesenchymal cells. Microvasc Res. 2013;86:21-9.

14 Galipeau J, Sensébé L. Mesenchymal stromal cells: clinical challenges and therapeutic opportunities. Cell Stem Cell. 2018;22(6):82433.

15 Hoban DB, Howard L, Dowd E. GDNF-secreting mesenchymal stem cells provide localized neuroprotection in an inflammationdriven rat model of Parkinson's disease. Neuroscience. 2015 Sep 10;303:402-11.

16 Guescini M, Genedani S, Stocchi V, Agnati LF. Astrocytes and glioblastoma cells release exosomes carrying mtDNA. J Neural Transm. 2010 Jan;117(1):1-4.

17 Thakur BK, Zhang H, Becker A, Matei I, Huang Y, Costa-Silva B, et al. Double-stranded DNA in exosomes: a novel biomarker in cancer detection. Cell Res. 2014 Jun;24(6): 766-9.

18 Skotland T, Sandvig K, Llorente A. Lipids in exosomes: current knowledge and the way forward. Prog Lipid Res. 2017 Apr;66:30-41.

19 Liu W, Wang Y, Gong F, Rong Y, Luo Y, Tang $\mathrm{P}$, et al. Exosomes derived from bone mesenchymal stem cells repair traumatic spinal cord injury by suppressing the activation of $\mathrm{A} 1$ neurotoxic reactive astrocytes. J Neurotrauma. 2019 Feb 1;36(3):469-84.

20 Reza-Zaldivar EE, Hernandez-Sapiens MA, Gutierrez-Mercado YK, Sandoval-Avila S, Gomez-Pinedo U, Marquez-Aguirre AL, et al. Mesenchymal stem cell-derived exosomes promote neurogenesis and cognitive function recovery in a mouse model of Alzheimer's disease. Neural Regen Res. 2019 Sep;14(9): 1626-34.

21 Pegtel DM, Peferoen L, Amor S. Extracellular vesicles as modulators of cell-to-cell communication in the healthy and diseased brain. Philos Trans R Soc Lond B Biol Sci. 2014 Sep 26;369(1652):20130516.

22 Mundra V, Gerling IC, Mahato RI. Mesenchymal stem cell-based therapy. Mol Pharm. 2013 Jan 7;10(1):77-89.

23 Rasmusson I, Ringdén O, Sundberg B, Le Blanc K. Mesenchymal stem cells inhibit the formation of cytotoxic $\mathrm{T}$ lymphocytes, but not activated cytotoxic $\mathrm{T}$ lymphocytes or natural killer cells. Transplantation. 2003 Oct; 76(8):1208-13

24 Sotiropoulou PA, Perez SA, Gritzapis AD, Baxevanis CN, Papamichail M. Interactions between human mesenchymal stem cells and natural killer cells. Stem Cells. 2006;24(1):7485.
25 Mehdipour F, Razmkhah M, Rezaeifard S, Bagheri M, Talei AR, Khalatbari B, et al. Mesenchymal stem cells induced anti-inflammatory features in B cells from breast tumor draining lymph nodes. Cell Biol Int. 2018;42(12):165869.

26 Gronthos S, Zannettino AC, Hay SJ, Shi S, Graves SE, Kortesidis A, et al. Molecular and cellular characterisation of highly purified stromal stem cells derived from human bone marrow. J Cell Sci. 2003 May 1;116(Pt 9): 1827-35.

27 Yun HM, Kim HS, Park KR, Shin JM, Kang $A R$, il Lee K, et al. Placenta-derived mesenchymal stem cells improve memory dysfunction in an A $\beta 1-42$-infused mouse model of Alzheimer's disease. Cell Death Dis. 2013 Dec 12;4(12):e958.

28 Eggenhofer E, Renner P, Soeder Y, Popp FC, Hoogduijn MJ, Geissler EK, et al. Features of synergism between mesenchymal stem cells and immunosuppressive drugs in a murine heart transplantation model. Transpl Immunol. 2011;25(2-3):141-7.

29 Song Y, Wang Z, Wang Z, Zhang H, Li X, Chen B. Use of FK506 and bone marrow mesenchymal stem cells for rat hind limb allografts. Neural Regen Res. 2012;7(34):2681-

30 Hajkova M, Hermankova B, Javorkova E, Bohacova P, Zajicova A, Holan V, et al. Mesenchymal stem cells attenuate the adverse effects of immunosuppressive drugs on distinct $\mathrm{T}$ cell subopulations. Stem Cell Rev Rep. 2017; 13(1):104-15

31 Hajkova M, Javorkova E, Zajicova A, Trosan P, Holan V, Krulova M. A local application of mesenchymal stem cells and cyclosporine A attenuates immune response by a switch in macrophage phenotype. J Tissue Eng Regen Med. 2017;11(5):1456-65

32 Lang AE, Lozano AM. Parkinson's disease. Second of two parts. N Engl J Med. 1998; 339(16):1130-43.

33 Sulzer D, Alcalay RN, Garretti F, Cote L, Kanter E, Agin-Liebes J, et al. T cells from patients with Parkinson's disease recognize alpha-synuclein peptides. Nature. 2017 Jun 29; 546(7660):656-61.

34 Mostafavi H, Ghassemifard L, Rostami A, Alipour M, Nadri S. Trabecular meshwork mesenchymal stem cell transplantation improve motor symptoms of parkinsonian rat model. Biologicals. 2019 Sep;61:61-7.

35 Cova L, Armentero MT, Zennaro E, Calzarossa C, Bossolasco P, Busca G, et al. Multiple neurogenic and neurorescue effects of human mesenchymal stem cell after transplantation in an experimental model of Parkinson's disease. Brain Res. 2010 Jan 22;1311:12-27.

36 Park HJ, Oh SH, Kim HN, Jung YJ, Lee PH. Mesenchymal stem cells enhance alpha-synuclein clearance via M2 microglia polarization in experimental and human parkinsonian disorder. Acta Neuropathol. 2016 Nov; 132(5):685-701.
37 Ge L, Jiang M, Duan D, Wang Z, Qi L, Teng $\mathrm{X}$, et al. Secretome of olfactory mucosa mesenchymal stem cell, a multiple potential stem cell. Stem Cells Int. 2016;2016:1243659.

38 Venkataramana NK, Kumar SKV, Balaraju S, Radhakrishnan RC, Bansal A, Dixit A, et al. Open-labeled study of unilateral autologous bone-marrow-derived mesenchymal stem cell transplantation in Parkinson's disease. Transl Res. 2010;155(2):62-70.

39 Berchtold NC, Cotman CW. Evolution in the conceptualization of dementia and Alzheimer's disease: Greco-Roman period to the 1960s. Neurobiol Aging. 1998 May-Jun;19(3): $173-89$.

40 Aisen PS, Cummings J, Jack CR Jr, Morris JC, Sperling R, Frölich L, et al. On the path to 2025: understanding the Alzheimer's disease continuum. Alzheimers Res Ther. 2017;9(1): 60.

41 Wu H-Y, Hudry E, Hashimoto T, Kuchibhotla K, Rozkalne A, Fan Z, et al. Amyloid $\beta$ induces the morphological neurodegenerative triad of spine loss, dendritic simplification, and neuritic dystrophies through calcineurin activation. J Neurosci. 2010;30(7):2636-49.

42 Lee JK, Jin HK, Bae JS. Bone marrow-derived mesenchymal stem cells reduce brain amyloid-beta deposition and accelerate the activation of microglia in an acutely induced Alzheimer's disease mouse model. Neurosci Lett. 2009;450(2):136-41.

43 Jiao H, Shi K, Zhang W, Yang L, Yang L, Guan $\mathrm{F}$, et al. Therapeutic potential of human amniotic membrane-derived mesenchymal stem cells in APP transgenic mice. Oncol Lett. 2016;12(3):1877-83.

44 Ma T, Gong K, Ao Q, Yan Y, Song B, Huang $\mathrm{H}$, et al. Intracerebral transplantation of adipose-derived mesenchymal stem cells alternatively activates microglia and ameliorates neuropathological deficits in Alzheimer's disease mice. Cell Transplant. 2013;22(Suppl 1): S113-26.

45 Park D, Yang G, Bae DK, Lee SH, Yang YH, Kyung J, et al. Human adipose tissue-derived mesenchymal stem cells improve cognitive function and physical activity in ageing mice. J Neurosci Res. 2013;91(5):660-70.

$46 \mathrm{Wu}$ C-C, Wang IF, Chiang PM, Wang LC, Shen CKJ, Tsai KJ. G-CSF-mobilized bone marrow mesenchymal stem cells replenish neural lineages in Alzheimer's disease mice via CXCR4/SDF-1 chemotaxis. Mol Neurobiol. 2017;54(8):6198-212.

47 Farahzadi R, Fathi E, Vietor I. Mesenchymal stem cells could be considered as a candidate for further studies in cell-based therapy of Alzheimer's disease via targeting the signaling pathways. ACS Chem Neurosci. 2020;11(10): 1424-35.

48 Blasco H, Mavel S, Corcia P, Gordon PH. The glutamate hypothesis in ALS: pathophysiology and drug development. Current Med Chem. 2014;21(31):3551-75. 
49 Cleveland DW, Rothstein JD. From Charcot to Lou Gehrig: deciphering selective motor neuron death in ALS. Nat Rev Neurosci. 2001 Nov;2(11):806-19.

50 Mazzini L, Vescovi A, Cantello R, Gelati M, Vercelli A. Stem cells therapy for ALS. Expert Opin Biol Ther. 2016;16(2):187-99.

51 Bonafede R, Scambi I, Peroni D, Potrich V, Boschi F, Benati D, et al. Exosome derived from murine adipose-derived stromal cells: neuroprotective effect on in vitro model of amyotrophic lateral sclerosis. Exp Cell Res. 2016 Jan 1;340(1):150-8.
52 Lee M, Ban JJ, Kim KY, Jeon GS, Im W, Sung JJ, et al. Adipose-derived stem cell exosomes alleviate pathology of amyotrophic lateral sclerosis in vitro. Biochem Biophys Res Commun. 2016 Oct 21;479(3):434-9.

53 Petrou P, Gothelf Y, Argov Z, Gotkine M, Levy YS, Kassis I, et al. Safety and clinical effects of mesenchymal stem cells secreting neurotrophic factor transplantation in patients with amyotrophic lateral sclerosis: results of phase $1 / 2$ and 2a clinical trials. JAMA Neurol. 2016 Mar;73(3):337-44.
54 Ferrero I, Mazzini L, Rustichelli D, Gunetti $\mathrm{M}$, Mareschi K, Testa L, et al. Bone marrow mesenchymal stem cells from healthy donors and sporadic amyotrophic lateral sclerosis patients. Cell Transplant. 2008;17(3):255-66.

55 Rajabzadeh N, Fathi E, Farahzadi R. Stem cellbased regenerative medicine. Stem Cell Investig. 2019;6:19.

56 Kabat M, Bobkov I, Kumar S, Grumet M. Trends in mesenchymal stem cell clinical trials 2004-2018: Is efficacy optimal in a narrow dose range? Stem Cells Transl Med. 2020; 9(1):17-27.

57 Yin JQ, Zhu J, Ankrum JA. Manufacturing of primed mesenchymal stromal cells for therapy. Nat Biomed Eng. 2019;3(2):90-104. 